\title{
CrystEngComm
}

www.rsc.org/crystengcomm
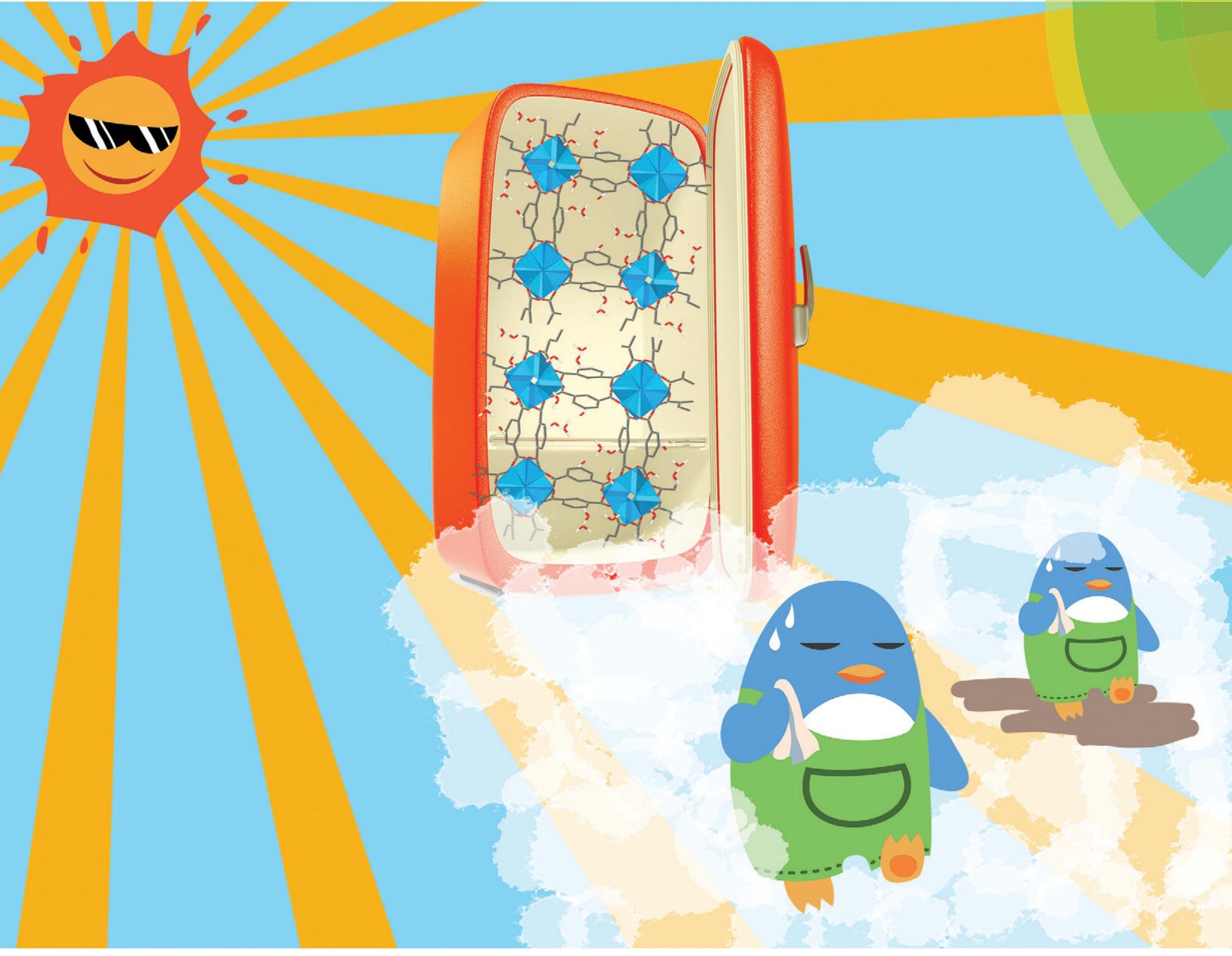


\title{
CrystEngComm
}

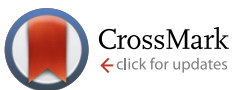

Cite this: CrystEngComm, 2015, 17, 5911

Received 22nd April 2015, Accepted 12th May 2015

DOI: 10.1039/c5ce00789e

www.rsc.org/crystengcomm

\section{Manufacture of dense CAU-10-H coatings for application in adsorption driven heat pumps: optimization and characterization $\dagger$}

\author{
M. F. de Lange, ${ }^{\star a b}$ T. Zeng, ${ }^{\text {ac }}$ T. J. H. Vlugt, ${ }^{b}$ J. Gascon ${ }^{a}$ and F. Kapteijn*a
}

\begin{abstract}
CAU-10-H displays a highly suitable step-wise water adsorption behaviour for application in adsorption driven heat pumps and chillers. For actual application, manufacturing of coatings of this material on thermally conductive surfaces is highly desired. Direct, single-step, crystallization of CAU-10-H on either metallic or anodized aluminium yields a significant amount of by-product(s) and an inhomogeneous substrate coverage. Although adequate pretreatment of the substrates before crystallization improves the quality of obtained coatings, significant improvements are achieved only when crystal nucleation and growth are separated. More specifically, application of a reactive seeding approach with anodized aluminium leads to full coverage of the substrate surface, high MOF loading and high purity of the crystalline phase, homogeneous layer thickness, narrow crystal size distribution, and good stability in repeated water adsorptiondesorption cycles.
\end{abstract}

\section{Introduction}

Adsorption driven heat pumps and chillers, AHP/ADC's, have great potential for reducing primary energy consumption and mitigating associated $\mathrm{CO}_{2}$ emissions and anthropogenic climate change. ${ }^{1}$ Devices based on this principle can potentially employ low grade thermal energy, e.g. solar or industrial waste heat, to sustainably supply cooling and heating, making use of the reversible ad- and desorption of, preferably, water. Compared to commercially applied adsorbents, the MetalOrganic Framework (MOF) CAU-10-H shows a higher volumetric adsorption capacity and thermodynamic efficiency for water as a working fluid. ${ }^{1,2}$ CAU-10-H contains isophthalic acid as organic linker and cis-connected $\mathrm{AlO}_{6}$-polyhedra, forming helical chains. The resulting structure has unidirectional pores. This MOF consists of abundantly available aluminium and isophthalic acid, both of which are produced industrially on a large scale, ${ }^{3}$ placing this MOF among the most commercially viable ones. Furthermore, its synthesis

\footnotetext{
${ }^{a}$ Catalysis Engineering, Chemical Engineering Department, Delft University of Technology, Julianalaan 136, 2628 BL Delft, The Netherlands. E-mail:m.f.delange@tudelft.nl, f.kapteijn@tudelft.nl

${ }^{b}$ Engineering Thermodynamics, Process \& Energy Laboratory, Delft University of Technology, Leeghwaterstraat 39, 2628 CB Delft, The Netherlands

${ }^{c}$ Department Thermo-chemical Conversion, Deutsches Biomasse-

forschungszentrum gemeinnützige GmbH, Torgauer Straße 116, 04347 Leipzig, Germany

$\dagger$ Electronic supplementary information (ESI) available: Characterization methods and additional characterization on powder samples, bare supports and coatings obtained using either direct synthesis, reactive or thermal seeding. See DOI: $10.1039 / \mathrm{c} 5 \mathrm{ce} 00789 \mathrm{e}$
}

does not require an expensive sacrificial template, in contrast to e.g. the zeotype structures of the AQSOA-series ${ }^{4-6}$ used in commercially available devices of Mitsubishi. ${ }^{7-13}$ The potential of CAU-10-H is further strengthened by the fact that the material is perfectly stable towards water and has not shown any sign of degradation over 700 repeated adsorption/desorption cycles, ${ }^{14}$ a feature not commonly encountered for MOFs when exposed to water. ${ }^{1}$ Altogether, these features confirm CAU-10-H as a commercially viable adsorbent for application in adsorption driven heat pumps and chillers. For successful implementation however, heat and mass transport should be fast enough to allow for high volume-specific power output. A promising way to achieve this is by coating a thermally conductive surface with the adsorbent of choice. E.g., the AQSOAseries are coated on a heat exchanger by using a binder. ${ }^{6,7}$ Furthermore, as mentioned by Ferey et al., MOFs show improved efficiency for air dehumidification, an application that shows similarities with AHP/ADC's, compared to zeolitic materials, when they are coated on honeycomb shaped rotors. ${ }^{15}$ To create such a coating, ideally, a binderless method is preferred as the binder does not only add to the cost of a device, but also dilutes the active material, decreasing the overall efficiency of the system. When MOFs are considered specifically, (organic) binders cannot be removed by combustion after coating, as this would also cause oxidation of the ligand of the MOF. Therefore, a coating by directly crystallizing the MOF on a thermally conductive surface is preferred. ${ }^{16}$ Previous results already indicated that CAU-10-H coatings can be formed directly on metallic aluminium (m-Al) supports. ${ }^{17}$ By addition of $\mathrm{HCl}$ to the reaction mixture, a 
higher surface loading was achieved, but an incomplete surface coverage and a broad range of crystal sizes were observed. Furthermore, $\mathrm{HCl}$ addition induced the formation of unidentified by-product(s). ${ }^{17}$ The aim of this study is to optimize the properties of the formed crystalline layer of CAU-10-H on aluminium substrates. Desired properties for this layer are: (i) full coverage of the surface, (ii) high MOF loading, (iii) homogeneous layer thickness, (iv) narrow crystal size distribution, (v) sufficient stability under working conditions and (vi) high purity of the crystalline phase. Here results of a systematic study of different synthesis parameters and an optimized protocol for the synthesis of homogeneous CAU-10$\mathrm{H}$ coatings are presented.

\section{Experimental}

\section{Materials}

Two different types of substrates were applied. Metallic aluminium (m-Al) substrates with a thickness of $0.5 \mathrm{~mm}$ and a purity of 99.9999\% were purchased from Mateck GmbH. Anodized aluminium (a-Al) substrates (Durapor 15) with a thickness of $0.5 \mathrm{~mm}$ were purchased from Polychromal B.V. According to the manufacturer's specification, the aluminium oxide layer is $15 \mu \mathrm{m}$ thick. For all syntheses, substrates were cut into squares of $20 \times 20 \mathrm{~mm}$ with corners cut-off. Typically, the sample mass was $\sim 0.54 \mathrm{~g}$ and $\sim 0.58 \mathrm{~g}$ for metallic and anodized aluminium substrates, respectively. The substrates were used either as received or after specified pretreatment. Isophthalic acid, 1,3- $\mathrm{H}_{2} \mathrm{BDC}(99 \%)$, aluminium sulfate octadecahydrate, $\mathrm{Al}_{2}\left(\mathrm{SO}_{4}\right)_{3} \cdot 18 \mathrm{H}_{2} \mathrm{O}$ (ACS reagent $\geq$ 99.8\%), $N, N$-dimethylformamide for synthesis, DMF (anhydrous $99.8 \%$ ), $N, N$-dimethylformamide for post-processing, DMF (puriss p.a., ACS reagent $\geq 99.8 \%$ ), hydrochloric acid, $\mathrm{HCl}$ (ACS reagent, 37\% in water), acetone (ACS reagent, $\geq$ 99.5\%) and methanol (anhydrous 99.8\%) were purchased from Sigma-Aldrich and used without further purification. Deionized water with a conductivity of $0.12 \mu \mathrm{S} \mathrm{cm}^{-1}$ was prepared using a MILIPORE MILI-Q.

\section{Powder synthesis CAU-10-H}

CAU-10-H powder was synthesized using two different ways, by conventional heating and microwave irradiation. The main difference in synthesis protocols lies in the employed reaction time, which is generally significantly shorter when microwave irradiation is used..$^{18-20}$ The molar ratios of reactants are identical in both approaches, as is the sample postprocessing.

Conventional synthesis. CAU-10-H was synthesized according to Reinsch et al. ${ }^{2} 1,3-\mathrm{H}_{2} \mathrm{BDC}(1.0 \mathrm{mmol}, 0.16 \mathrm{~g})$, $\mathrm{Al}_{2}\left(\mathrm{SO}_{4}\right)_{3} \cdot 18 \mathrm{H}_{2} \mathrm{O}$ (1.0 mmol, $0.64 \mathrm{~g}$ ), DMF (10.6 mmol, $0.76 \mathrm{~g}$ ) and deionized water $(3.3 \mathrm{ml})$ were added to a Teflon insert with a capacity of $45.0 \mathrm{ml}$. The Teflon insert was closed with a lid, sealed in an autoclave, and heated in a convection oven (Heraeus $\mathrm{T} 6,5{ }^{\circ} \mathrm{C} \mathrm{min}^{-1}$ ) to the required reaction temperature $\left(135^{\circ} \mathrm{C}\right)$, left for $12 \mathrm{~h}$ and then allowed to cool to ambient temperature. Stirring was not applied during synthesis.
Microwave synthesis. 1,3- $\mathrm{H}_{2} \mathrm{BDC}(12.9 \mathrm{mmol}, 2.1 \mathrm{~g})$, $\mathrm{Al}_{2}\left(\mathrm{SO}_{4}\right)_{3} \cdot 18 \mathrm{H}_{2} \mathrm{O}$ (12.9 mmol, $\left.8.6 \mathrm{~g}\right)$, DMF (137 mmol, $10.0 \mathrm{~g}$ ) and deionized water $(42.4 \mathrm{ml})$ were added to a Teflon insert with a capacity of $90 \mathrm{ml}$. The vessel was sealed, equipped with a thermocouple and placed in the microwave oven (Milestone MultiSYNTH, $300 \mathrm{~W}, 10{ }^{\circ} \mathrm{C} \mathrm{min}^{-1}$ ). The reaction mixture was heated to the required reaction temperature (135 ${ }^{\circ} \mathrm{C}$ ), left for $1 \mathrm{~h}$ at this temperature and then allowed to cool to ambient temperature. Stirring was not applied during synthesis.

Post-processing (powder). After synthesis and cooling to ambient temperature, the reaction mixture was filtered using a diaphragm vacuum pump (Vacuubrand MZ 2C, $1.7 \mathrm{~m}^{3} \mathrm{~h}^{-1}$ ), a Büchner funnel with filtration paper (GVS Maine Magna, nylon membrane filter, type: plain, pore size $0.45 \mu \mathrm{m}$, diameter $90 \mathrm{~mm}$ ) and a side arm filtering flask. The loaded filter paper was dried in a muffle oven at $100{ }^{\circ} \mathrm{C}$ for about $20 \mathrm{~min}$. The filtration residue was transferred from the filtration paper into a beaker where it was submerged in about $20 \mathrm{ml}$ DMF at room temperature overnight. The suspension was again filtered and dried as above. The filtration residue was then submerged in about $20 \mathrm{ml}$ deionised water at room temperature overnight. After filtration and drying, the powder was stored in a sample vial.

\section{Formation of CAU-10-H on substrates}

Three synthesis protocols have been followed in order to optimize the formation of CAU-10-H on aluminium substrates: (i) the single-step direct synthesis, adopted from previous work, ${ }^{17}$ and two multi-step procedures in order to separate crystal nucleation and growth, (ii) reactive ${ }^{21}$ and (iii) thermal seeding. ${ }^{22}$ All substrates and possible solids obtained in the reaction solution (i.e. filtration residue) were activated following an identical protocol.

Substrate pretreatment. Pretreatment of as-received substrates might be necessary to remove surface impurities, and furthermore to enhance reactivity of the substrate before synthesis. To this end, two different substrate pretreatment methods have been applied, based on previously reported procedures by Arnold et al. ${ }^{23}$ and Bux et al. ${ }^{24}$ Method A comprises only cleaning in solvents, while method B also includes a mild acid treatment with $\mathrm{HCl}$. Details can be found in the ESI† (section S1).

Direct synthesis coating. In attempts to optimize the quality of CAU-10-H coatings on aluminium-supports, process parameters and synthesis mixture compositions were varied systematically. Starting point is the synthesis protocol found most successful in previous studies, ${ }^{17}$ for both metallic (m-Al) and anodized (a-Al) aluminium substrates, either pre-treated or as received. A given substrate was placed in a Teflon insert with a capacity of $45.0 \mathrm{ml}$. Subsequently, the reaction mixture was added to the insert. Standard composition is $1,3-\mathrm{H}_{2} \mathrm{BDC}$ (5.2 mmol, $0.86 \mathrm{~g})$, deionized water $(17.0 \mathrm{ml})$, DMF $(4.2 \mathrm{ml})$ and $\mathrm{HCl}_{\mathrm{aq}}(1.7 \mathrm{ml}, 37 \%$ in water). The Teflon insert was then closed with a lid, sealed in an autoclave, and heated to the 
required reaction temperature in a convection oven (Heraeus T6, $5{ }^{\circ} \mathrm{C} \mathrm{min}^{-1}$ ) for a given reaction time. For the standard synthesis protocol, reaction temperature is $135^{\circ} \mathrm{C}$ and reaction time is $12 \mathrm{~h}$. This, in combination with the abovementioned standard synthesis mixture composition, will be referred to as "standard synthesis protocol" (SSP). Using these conditions as starting point, e.g. the amount of added DMF was reduced $(75 \%, 50 \%, 25 \%, 0 \%)$, the amount of $\mathrm{HCl}$ was varied $(200 \%, 50 \%, 0 \%)$, an additional aluminium source $\left(\mathrm{Al}_{2}\left(\mathrm{SO}_{4}\right)_{3} \cdot 18 \mathrm{H}_{2} \mathrm{O}\right)$ was added (up to $3 \mathrm{~g}$ ), and reaction time was altered, one variation at a time. When the amount of $\mathrm{HCl}$ solution is altered, the amount of deionized water is adjusted to keep the total amount of water constant. After reaction, the autoclave was removed from the oven and allowed to cool to ambient temperature. The reaction mixture including the substrate was subjected to post-processing (vide infra).

Reactive seeding coating. The reactive seeding approach is based on the methodology applied by $\mathrm{Hu}$ et al., who used it to create MIL-53(Al) membranes on alumina supports. ${ }^{21}$ The approach revolves around two distinct steps. In the first, small MOF crystals (seeds) are attached to the surface via a synthetic reaction. In the second, these seeds are grown to large crystals in the presence of MOF synthesis precursors under hydrothermal conditions.

For the reactive seeding step, pretreated substrates were placed in a Teflon insert with a capacity of $45.0 \mathrm{ml}$. Subsequently, 1,3- $\mathrm{H}_{2}$ BDC (5.2 mmol, $\left.0.86 \mathrm{~g}\right)$, DMF (4.2 ml), $\mathrm{HCl}$ (37\% in water, $1.7 \mathrm{ml})$ and deionized water $(17.0 \mathrm{ml})$ were added. For a-Al, no $\mathrm{HCl}$ was added and the amount of deionized water was adjusted to keep the total amount of water constant. The Teflon insert was closed with a lid, sealed in an autoclave, and heated in a convection oven (Heraeus T6, 5 $\left.{ }^{\circ} \mathrm{C} \min ^{-1}\right)$ to the required reaction temperature $\left(135^{\circ} \mathrm{C}\right)$. Stirring was not applied during synthesis. The time allowed for reactive seeding, $t_{\mathrm{rs}}$ was varied from 1 to $4 \mathrm{~h}$. After reaction, the autoclave was removed from the oven and allowed to cool to ambient temperature. The seeded substrate was thoroughly rinsed with DMF and deionized water and subsequently dried in an oven (Heraeus, T5042) at $100{ }^{\circ} \mathrm{C}$ for $1 \mathrm{~h}$. This seeded substrate was weighed and stored in a sample vial, until used in the second step.

For secondary growth, the seeded substrates were placed once more in a Teflon insert with a capacity of $45.0 \mathrm{ml}$. Either of two different precursor solutions, without added acidity, were used keeping volume of water constant, employing a dilution ratio (DR) of either 2 or 5 for the other reactants, relative to conventional synthesis. E.g. for a dilution ratio of 2 , the following was added: $1,3-\mathrm{H}_{2} \mathrm{BDC}$ (2.6 mmol, $0.43 \mathrm{~g}$ ), $\mathrm{Al}_{2}\left(\mathrm{SO}_{4}\right)_{3} \cdot 18 \mathrm{H}_{2} \mathrm{O}$ (2.6 mmol, $1.7 \mathrm{~g}$ ), DMF (2.2 $\mathrm{ml})$, deionized water $(17.0 \mathrm{ml})$. For a ratio of 5 , this becomes: 1,3- $\mathrm{H}_{2} \mathrm{BDC}(1.0 \mathrm{mmol}, 0.17 \mathrm{~g}), \mathrm{Al}_{2}\left(\mathrm{SO}_{4}\right)_{3} \cdot 18 \mathrm{H}_{2} \mathrm{O}(1.0 \mathrm{mmol}$, $0.69 \mathrm{~g})$, DMF $(0.84 \mathrm{ml}, 0.80 \mathrm{~g})$, deionized water $(17.0 \mathrm{ml})$. The Teflon insert was closed with a lid, sealed in an autoclave, and heated in a convection oven (Heraeus $\mathrm{T} 6,5^{\circ} \mathrm{C} \mathrm{min}^{-1}$ ) to the required reaction temperature $\left(135^{\circ} \mathrm{C}\right)$. After a reaction time of $12 \mathrm{~h}$, the autoclave was removed from the oven and allowed to cool to ambient temperature. The reaction mixture with the substrate was post-processed as described previously.

Thermal seeding coating. The thermal seeding approach is adapted from Guerrero et al., who applied this method for the creation of HKUST-1 membranes on porous supports. ${ }^{22}$ The procedure consists of three steps: seed formation, attachment of seeds to the substrate surface and secondary growth.

The used seeds are created with microwave heating. Three different seed solutions are employed. Solution 1 is the reaction mixture, after cooling down and without further treatment (for a yield of $\sim 65 \%$, as commonly observed for conventional synthesis, ${ }^{2}$ this would be roughly 3 wt.\% CAU-10-H). For the other solutions, CAU-10-H powder was processed after synthesis as before. Seed solutions 2 and 3 contain CAU-10-H seeds dispersed in deionized water, respectively with 2.5 and 5 wt.\% MOF. Prior to the seeding experiments, the selected seed solution was sonicated in an ultrasonic bath (VGT-1730QT, $100 \mathrm{~W}, 40 \mathrm{kHz}$ ) for $1 \mathrm{~min}$ to break down agglomerates.

For the attachment of seeds, pretreated substrates were placed in a petri dish and heated in a convection oven (Heraeus T6, $5^{\circ} \mathrm{C} \mathrm{min}^{-1}$ ) at $200{ }^{\circ} \mathrm{C}$ for $15 \mathrm{~min}$. While the substrates were still inside the oven, a seed solution was dropped on the surface of the hot substrates using a pipette until the surface was completely covered with the solution $(2-6 \mathrm{ml}$, roughly). The substrates were kept inside the oven for $15 \mathrm{~min}$ to allow for complete evaporation of the solvent. To obtain a complete coverage of the substrate surface with seed crystals, this procedure had to be repeated two more times when seed solution 1 was used. In contrast, only one thermal seeding step was required when either solution 2 or 3 , only containing CAU-10-H seeds in water, was used. The seeded substrates were then rinsed with deionized water, to remove excess material and not attached seeds, and subsequently dried in an oven (Heraeus T5042) at $100{ }^{\circ} \mathrm{C}$ for $1 \mathrm{~h}$. The secondary growth step is the same for thermal and reactive seeding (vide supra).

Post-processing. After completion of a coating synthesis, the reaction mixture was allowed to cool to ambient temperature. Subsequently, this mixture was filtered using a diaphragm vacuum pump (Vacuubrand $\mathrm{MZ} 2 \mathrm{C}, 1.7 \mathrm{~m}^{3} \mathrm{~h}^{-1}$ ), a Büchner funnel with filtration paper (GVS Maine Magna, nylon membrane filter, type: plain, pore size $0.45 \mu \mathrm{m}$, diameter $90 \mathrm{~mm}$ ) and a side arm filtering flask. After filtration, the substrate covered with MOF and the loaded filter paper were treated individually. The substrates were submerged in DMF overnight at room temperature. The solvent was decanted and the substrate was rinsed with deionized water and submerged in about $20 \mathrm{ml}$ deionized water overnight at room temperature. Afterwards, the water was decanted and the substrate was rinsed with deionized water and dried in a muffle oven at $100{ }^{\circ} \mathrm{C}$ overnight. After drying, the substrate was weighted and stored in a sample vial. The filtration residue was processed in the same way as regular powder samples. 
All methods and procedures for characterization of powder samples and substrates can be found in detail in section S2. $†$

\section{Results and discussion}

The results of bulk (powder) synthesis of CAU-10-H are discussed as reference in comparison with CAU-10-H coated on substrates. The crystals formed are used as seeds in the thermal seeding approach. Subsequently, coatings obtained by direct synthesis are discussed briefly. The main focus of this article, the benefits of employing two multi-step approaches, reactive and thermal seeding are discussed next. Finally, selected substrates are characterized and compared in detail.

\section{Powder synthesis}

From both conventional and microwave synthesis, pure CAU-10-H is obtained (Fig. S1, S2 $\dagger$ ). The adsorption capacity of $\mathrm{N}_{2}$ and $\mathrm{H}_{2} \mathrm{O}$ is seemingly somewhat larger for the material obtained by microwave synthesis (Fig. S3†). As the difference in $\mathrm{N}_{2}$ adsorption capacity (which is determined at significantly lower temperatures) is larger, this can be attributed to diffusional limitations in the material obtained from conventional synthesis. This can be further made plausible by the fact that for $\mathrm{N}_{2}$ the adsorption hysteresis is not fully closed at $p / p_{\mathrm{o}} \leq 0.4$ for the material from conventional synthesis (Fig. S3†), another indication for possible diffusional limitations. $^{25,26}$ The fact that microwave synthesis, on average, results in smaller particle sizes is commonly observed for MOF crystals. ${ }^{18-20,27,28}$ SEM images reveal that the size of the smaller crystals present in both samples is roughly of the same order of magnitude, but after conventional synthesis, more and larger crystal agglomerates exist, likely causing the observed diffusional limitations (Fig. S4†).

Water adsorption measured at two temperatures for CAU-10-H(MW), see Fig. 1, allows for the calculation of the isosteric enthalpy of adsorption. As previously noted, ${ }^{1,17}$ the reversible step in uptake, at $p / p_{\mathrm{o}} \sim 0.15$ makes CAU-10-H a suitable candidate for adsorption driven allocation of cold especially.

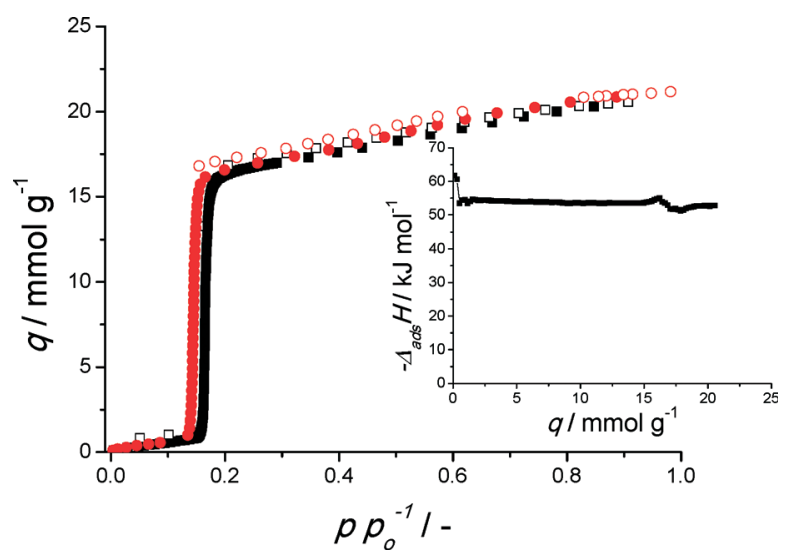

Fig. 1 Water adsorption isotherms of CAU-10-H obtained from microwave synthesis at $298(\square)$ and $288 \mathrm{~K}(\mathrm{O}$. Closed symbols indicate adsorption, open symbols desorption. (inset) Isosteric enthalpy of adsorption (calculated with eqn $(\mathrm{S} 1 \dagger)$ )).
The isosteric enthalpy of adsorption at the steep step in water uptake, $1<q<16 \mathrm{mmol} \mathrm{g}^{-1}$, is nearly constant (about $-53.5 \mathrm{~kJ}$ $\mathrm{mol}^{-1}$ ) and only slightly higher than the condensation enthalpy of water ( - 41 $\mathrm{kJ} \mathrm{mol}^{-1}$ at measurement temperature), making regeneration in adsorption driven heat pumps and chillers relatively energy efficient. ${ }^{1}$ Because only two isotherms were used, the uncertainty in the isosteric heat could not be calculated. However, with water as the adsorptive, this turns out to be only 3-4 $\mathrm{kJ} \mathrm{mol}^{-1}$, when additional isotherms are available. ${ }^{29}$

\section{Formation of CAU-10-H on substrates}

Support selection. In addition to the metallic aluminium (m-Al) substrates employed in previous studies, ${ }^{17}$ the effect of using anodized aluminium substrates having a porous (a-Al) layer has been investigated, since previous studies on porous $\gamma-\mathrm{Al}_{2} \mathrm{O}_{3}$ supports revealed that high loadings of CAU-10-H with homogeneous crystal size distributions could be obtained. ${ }^{17}$ As no aluminium-source is added to the synthesis mixture, crystal growth can only occur by extraction of Al-ions from the support. ${ }^{30}$ This process turned out to be more efficient for $\gamma$-aluminacontaining supports than for metallic aluminium. ${ }^{17}$

As metallic supports display higher thermal conductivity and are desired for the actual application, it was evident to employ aluminium supports with an (anodized) aluminiumoxide layer. The oxide layer is $15 \mu \mathrm{m}$ thick in case of a-Al, whereas on metallic aluminium a native oxidic skin layer of only $4 \mathrm{~nm}$ is present. $^{31}$ Furthermore, anodized oxide layers are composed of amorphous alumina, if not calcined thoroughly, ${ }^{32}$ which might further increase reactivity compared to $\gamma$-alumina. No XRD reflections were observed for the anodized layer itself (Fig. S5†), confirming the amorphous structure of the alumina. Because of the anodization, a-Al substrates display mesoporosity and have a small water adsorption capacity, features absent for m-Al (Fig. S6†).

Direct synthesis. Initial attempts at improving coatings were made by varying synthesis conditions. As these variations led to inferior coatings, these are discussed only briefly (details in section $\mathrm{S} 4 \dagger$ ). The addition of different amounts of $\mathrm{Al}_{2}\left(\mathrm{SO}_{4}\right)_{3} \cdot 18 \mathrm{H}_{2} \mathrm{O}$, the precursor used for bulk CAU-10-H, to the synthesis mixture (on $\mathrm{m}-\mathrm{Al}$ ) has an adverse effect on surface coverage, and the undesired secondary crystalline phase is more dominantly observed, a finding in concert with that of the work of Reinsch et al. for bulk powder synthesis. ${ }^{2}$

Reducing the amount of hydrochloric acid added by $50 \%$ for $\mathrm{m}$-Al (Fig. S9†) results in a slightly lower coverage. When the amount of $\mathrm{HCl}$ is doubled (Fig. S9†), coverage is greatly reduced and crystals apparently appear only along grooves, likely created by the preferential dissolution of $\mathrm{Al}^{3+}$ ions from local aluminium crystal boundaries in the metallic support, as was observed in a previous study. ${ }^{17}$ Using $\mathrm{HCl}$ for syntheses on a-Al is detrimental for the integrity of the support itself and leads to the formation of an amorphous phase (Fig. S10-S12 $\dagger$ ). The added isophthalic acid linker is thus more than sufficient to extract $\mathrm{Al}^{3+}$-ions from the support (a-Al), though $\mathrm{HCl}$ by-product formation and incomplete surface 
coverage are observed (Fig. S10, S12 $\dagger$ ). The utilization of additional acids thus is not necessary, having an adverse effect on the formation of MOF crystals (and will further not be used on a-Al substrates).

When the amount of DMF is significantly reduced (this has e.g. a positive effect on the crystallization of MIL-53(Al)- $\mathrm{NH}_{2}$ (ref. 33)) for syntheses on $\mathrm{m}-\mathrm{Al}$, no CAU-10-H is formed and instead a large amount of recrystallized isophthalic acid is found (Fig. S13-S15†). For a-Al, crystallization apparently becomes more homogeneous with decreasing amounts of DMF, though for all experiments large fractions of the crystals formed on the surface consist of by-product(s) (Fig. S14, S16 $\dagger$ ).

The reduction of temperature or prolongation of reaction time might yield more controlled crystal growth. ${ }^{34-37}$ Unfortunately, when synthesis is performed at a lower temperature $\left(115^{\circ} \mathrm{C}\right)$, no satisfactory crystal growth is achieved (Fig. S17†). Longer reaction times (at $135^{\circ} \mathrm{C}$ ) result in larger crystals on the surface (Fig. S18†), but coverage becomes more inhomogeneous and by-product formation is increased (Fig. S19†). Apparently, undesired Ostwald-ripening $^{38}$ occurs, limiting the use of longer crystallization times.

Support pretreatment. Pretreatment (methods A and B, vide supra) is used for two possible purposes here: (i) it might remove pollutants present on the substrate surface $23,39-41$ and (ii) it might create additional $\mathrm{OH}$-groups to improve reactivity. ${ }^{23,24,42,43}$ For m-Al substrates, surface coverage and crystal size distributions are hardly altered when either of the pretreatment methods is applied (Fig. S20†). For a-Al however, coverage is systematically increased from untreated to samples pretreated with method $\mathrm{A}$ and further with method B (Fig. S21†). This is likely due to impurities introduced during the anodization process, which are removed by pretreatment. For $\mathrm{m}-\mathrm{Al}$ method $\mathrm{A}$ is optimal, for a-Al this is method B (Fig. S22 $\dagger$ ), as by-product formation is lowest. The effect of pretreatment method A for m-Al and especially method $\mathrm{B}$ for a-Al, has a beneficial effect on reproducibility (Fig. S23-S27†). Again, when supports are pretreated, longer reaction times decrease the coating quality (Fig. S28-S32†).

In summary, using direct synthesis methods, it has been found nearly impossible to obtain coatings of improved quality by means of varying synthesis conditions. Therefore separating crystal nucleation and growth is concluded to be a more feasible strategy. To this end, the reactive and thermal seeding approaches, from $\mathrm{Hu}$ et $a ._{.}{ }^{21}$ and Guerrero et al., ${ }^{22}$ respectively, are adopted here.

Reactive seeding. To create small MOF crystals on the support, a synthesis between 1 and $4 \mathrm{~h}$ is performed on pretreated m-Al (method A) and pretreated a-Al (method B). Conditions are as defined (SSP, vide supra), though for a-Al $\mathrm{HCl}$ is omitted (SSPa). A seed reaction time of 1 or $2 \mathrm{~h}$ results in negligible coverage (Fig. S33 $\uparrow$ ). For reaction times of 3 and especially $4 \mathrm{~h}$, small crystals, seemingly CAU-10-H (Fig. S34 $\dagger$ ), can be observed on the surface of both substrates (Fig. 2, top). These seeded substrates are deemed suitable for secondary growth. When a dilution ratio of 2 is employed during secondary growth, incomplete and inhomogeneous substrate coverage is observed (a)
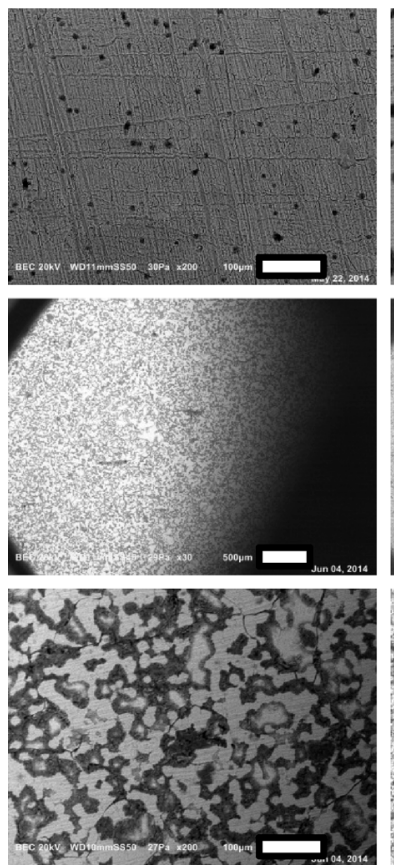

(b)
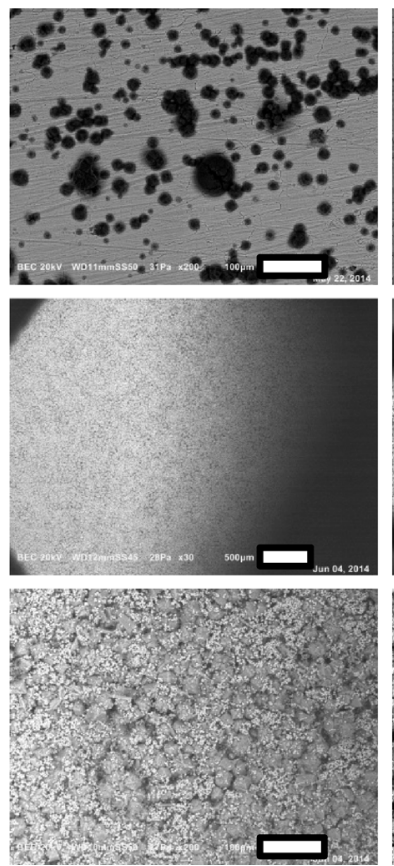

(c)
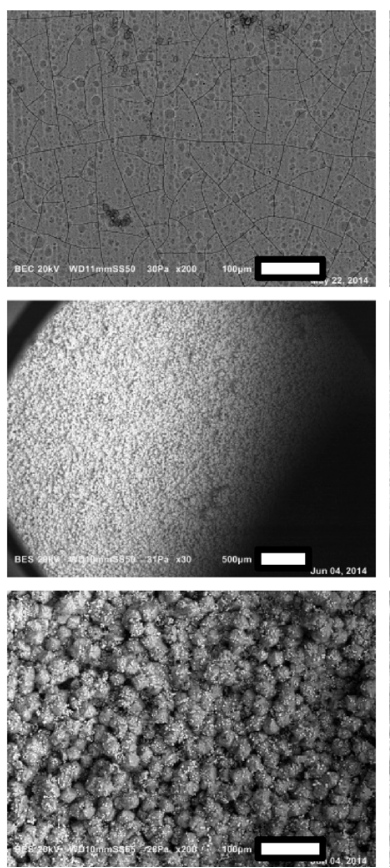

(d)
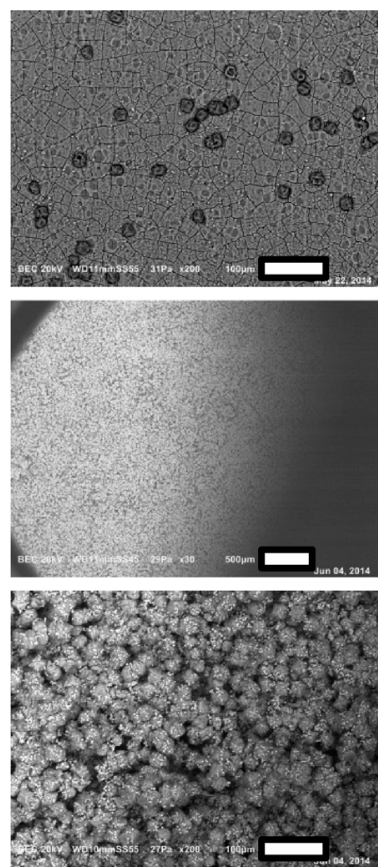

Fig. 2 SEM images of CAU-10-H synthesized by reactive seeding and secondary growth with a precursor dilution ratio of 5, for pretreated m-Al (method A) employing a reaction time for the seeding step of 3 (a) and 4 (b) h and for pretreated a-Al (method B), employing a reaction time for the seeding step of 3 (c) and 4 (d) h. Substrates after reactive seeding (top, scale bar represents $100 \mu \mathrm{m}$ ) and after secondary growth (middle, scale bar indicates $500 \mu \mathrm{m}$, bottom, scale bar represents $100 \mu \mathrm{m}$ ). 


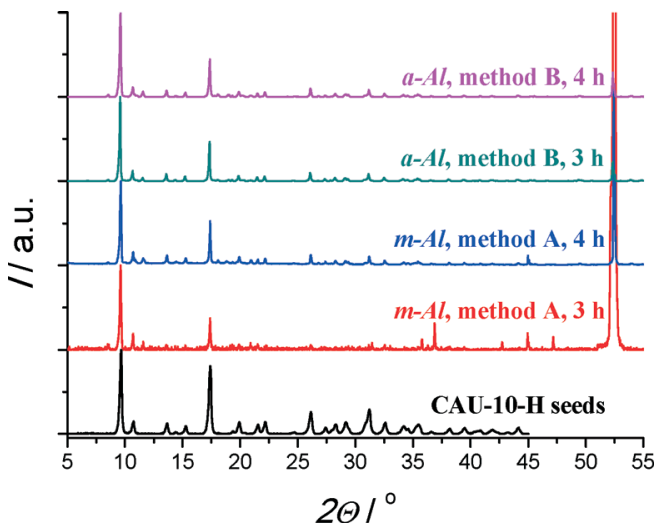

Fig. 3 XRD patterns of substrates after reactive seeding and secondary growth with a precursor dilution ratio of 5 , for pretreated $\mathrm{m}-\mathrm{Al}(\operatorname{method} \mathrm{A})$ and pretreated a-Al $(\operatorname{method} B)$, employing a reactive seeding time of 3 or $4 \mathrm{~h}$.

on both substrates (Fig. S35†). The crystals formed on the substrate surface seemingly are pure CAU-10-H for a-Al substrates (Fig. S36†), though for m-Al substrates unwanted by-product(s) are found. High purity CAU-10-H crystals are obtained in solution (Fig. S36†). These results clearly indicate that crystallization is too rapid when a dilution ratio of 2 is employed, resulting in detachment of CAU-10-H from supports and possibly facilitating nucleation and growth in the synthesis solution. Increasing the dilution ratio to 5 significantly increases coverage and homogeneity of the formed layers (based on SEM images, Fig. 2) and phase purity (Fig. 3).
Moreover, this is highly desired from a synthesis yield point of view, since most of the reactants are used to manufacture the coating, and the amount of detached crystals and unreacted reactants is minimized.

Remarkably, layers formed on anodized aluminium after 4 $\mathrm{h}$ of synthesis exhibit an excellent quality. A dense, homogeneous coverage of phase-pure CAU-10-H is created (Fig. 2d). The obtained quality is far better than that from syntheses performed in one step, indicating the potential of separating crystal nucleation and growth for the creation of MOF coatings on substrates.

Thermal seeding. The first step in thermal seeding is the formation of seeds synthesized via microwave heating (vide supra). Utilizing the solution obtained from microwave reaction directly (solution 1) fails to properly deposit seeds on either of the pretreated supports, even after repetitive addition of the seed solution (Fig. S37†). This is notably different for seed solutions 2 and 3, containing $2.5 \%$ and $5 \%$ wt. of CAU-10-H crystals dispersed in water, respectively, as seeds are clearly visible on the pretreated supports (Fig. 4, top). For secondary growth, only a dilution ratio of 5 has been employed, based on the experience with reactive seeding. The pretreated substrates after secondary growth show a satisfactory coverage (Fig. 4) and phase purity (Fig. 5).

Compared to the coverage obtained with reactive seeding, the crystal size distribution seems slightly less homogeneous and there appear to be some areas with lower coverage. This might be attributed to the less intimate contact of seeds with (a)
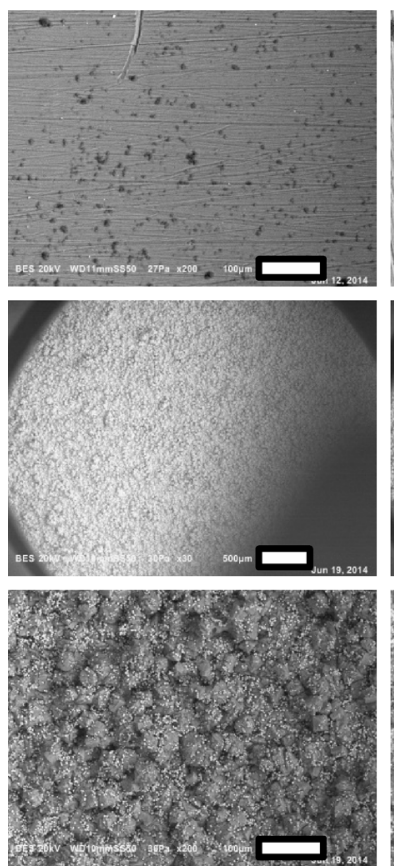

(b)
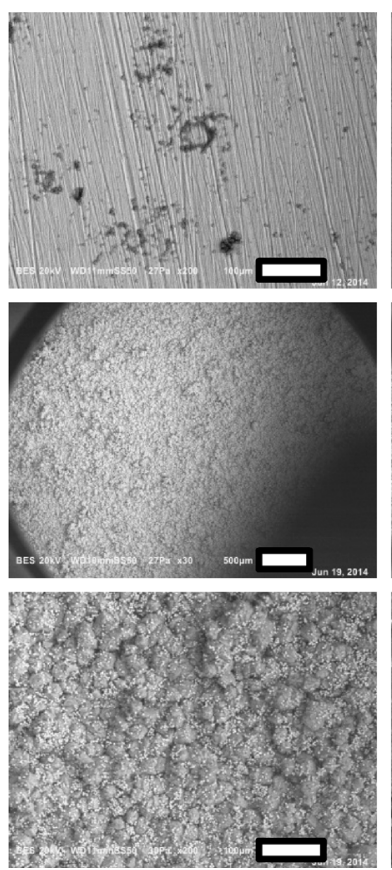

(c)
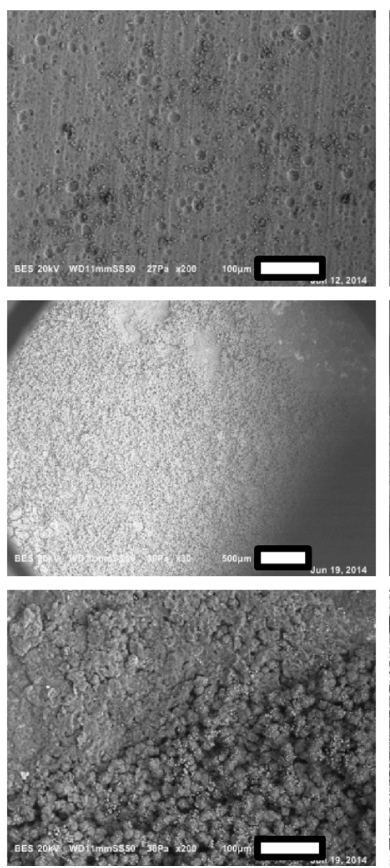

(d)
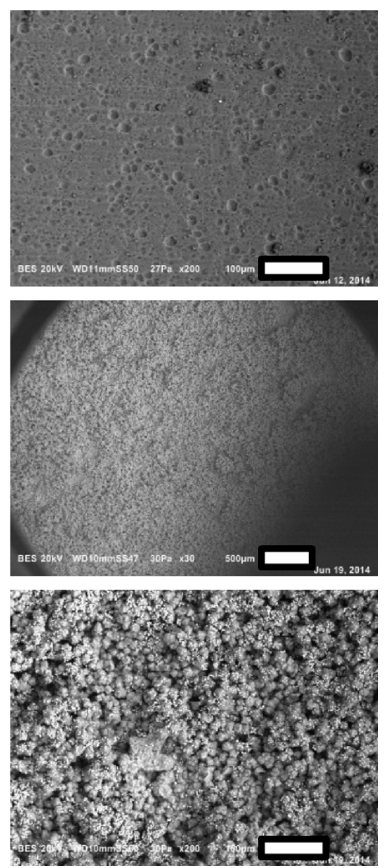

Fig. 4 SEM images of CAU-10-H synthesized by thermal seeding and secondary growth, for pretreated m-Al (method A), employing seed solution 2 (a) and 3 (b) and for pretreated a-Al (method B) employing seed solution 2 (c) and 3 (d). Substrates after thermal seeding and cleaning (top, scale bar indicates $100 \mu \mathrm{m}$ ) and after secondary growth, employing a dilution ratio of 5 (middle, scale bar indicates $500 \mu \mathrm{m}$, bottom, scale bar indicates $100 \mu \mathrm{m})$. 


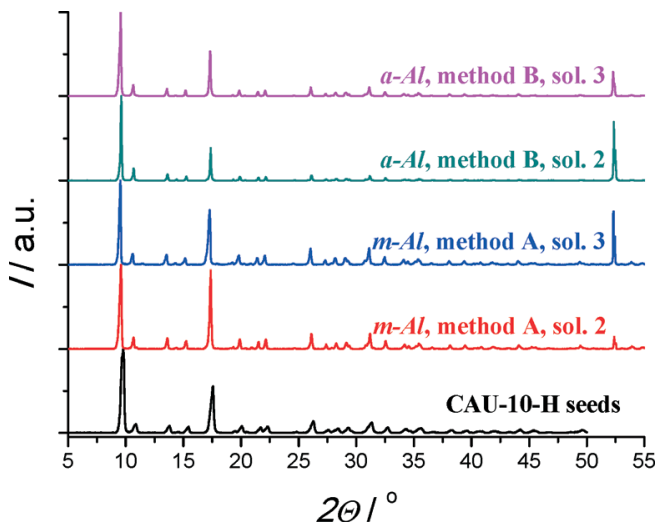

Fig. 5 XRD patterns of used $\mathrm{CAU}-10-\mathrm{H}$ seeds and substrates after thermal seeding and secondary growth, for pretreated $\mathrm{m}-\mathrm{Al}$ (method A) and pretreated a-Al (method B), using seed solution 2 (2.5 wt.\% seeds) and 3 (5 wt.\% seeds) and a dilution ratio of 5 .

the support in this method, than using reactive seeding. Nonetheless, also in this approach coverage, crystal homogeneity and phase purity are superior to those obtained via direct synthesis, once again underlining that separating crystal nucleation and growth is extremely suited for the creation of MOF coatings on substrates.

\section{Comparison of coated substrates}

Coating methods. In many of the performed syntheses, a significant amount of crystalline by-product is formed, of which the exact structure is not known. Recently it was reported that MIL-53(Al) is converted to non-porous $\gamma$ - $\mathrm{AlO}(\mathrm{OH})$ (boehmite) when exposed to water at elevated temperatures. ${ }^{44}$ Furthermore, under reaction conditions, insoluble $\mathrm{Al}(\mathrm{OH})_{3}$ might precipitate. ${ }^{45,46}$ Unfortunately, neither the XRD patterns of these phases, nor of those resulting from the recrystallization of any of the precursors correspond to the one of the formed by-product(s) (Fig. S38 $\dagger$ ). The same holds for either $\alpha$ - or $\gamma$-alumina. ${ }^{47}$ As the secondary phase(s) could not be isolated, the identity will remain unknown. However, compared to results obtained in previous work, ${ }^{17}$ significant advances have been made with respect to homogeneity in both substrate coverage and size distribution of the deposited CAU-10-H crystals. Furthermore, by-product formation is minimized when decoupling crystal growth and nucleation.

These advancements have been achieved by proper substrate pretreatment, employment of anodized alumina on the surface layer, and the separation of crystal nucleation and growth. Especially, reactive seeding on pretreated a-Al has been shown to be a promising route, based on SEM microscopy and X-ray diffraction patterns. These techniques however, do not yield any information on adsorption capacity and (cyclic) stability, nor on the chemical composition of the coating. To elucidate this, further characterization has been performed for a selection of samples. Specifically, CAU-10-H obtained by direct synthesis (DS.) on untreated (UT.) a-Al and $\mathrm{m}$-Al and on pretreated a-Al (method B, M.B.) and m-Al (method A, M.A.) are compared with the samples obtained by the reactive seeding (RS.) approach on both pretreated a-Al and $\mathrm{m}-\mathrm{Al}$.

IR spectra of CAU-10-H coated substrates after secondary synthesis (Fig. S40†) show similarity with the recorded spectrum for pure CAU-10-H (Fig. S39†), confirming once more the presence of CAU-10-H. However, there are notable differences as well, attributed primarily to the presence of byproduct(s). Comparison of these IR spectra (Fig. S39, S40†) tends to indicate that the by-product contains $\mathrm{Al}-\mathrm{OH}$ groups but hardly, if any, isophthalic acid (section S7†).

Thermo-gravimetric analysis (Fig. 6) is congruent with the qualitative indications by SEM analysis. The decrease in mass between 500 and $600{ }^{\circ} \mathrm{C}$, which represents exothermic oxidation of the organic ligand, is largest for reactive seeding on a-Al. This is also observed in the SDTA profile (Fig. S41†). Notable exception is the profile for the direct synthesis on pretreated a-Al, for which seemingly significant amounts of isophthalic acid are present (section S7†). Water adsorption isotherms on the selected coated substrates are shown in Fig. 7.

Clearly, for all but direct synthesis on untreated a-Al, the desired step of CAU-10-H (Fig. 1) is mimicked on these substrates at the same relative pressure, demonstrating that coatings indeed behave like the bulk material. The a-Al substrate coated with CAU-10-H by reactive seeding shows the highest water adsorption capacity, displaying a significantly improved capacity compared to the other substrates and previous work. ${ }^{17}$ Nitrogen adsorption isotherms reveal strong diffusional limitations and henceforth do not offer a solid basis for detailed interpretation (Fig. S42 $\dagger$ ).

In the preceding conclusions regarding purity, surface coverage and crystal size distribution and adsorption capacity have been drawn. Ideally one would like to supplement this with a quantification of the amount of CAU-10-H present for selected samples. This desire is not so easily fulfilled, as e.g. methods based on quantitative elemental analysis cannot differentiate between aluminium present in the support and in

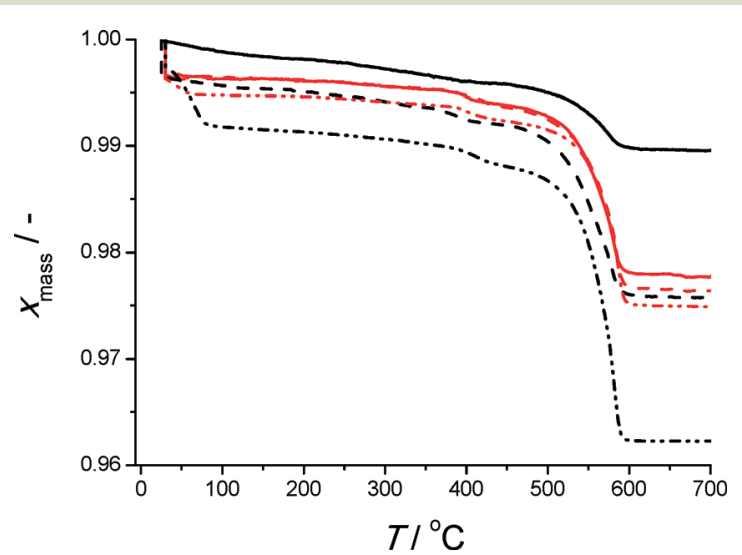

Fig. 6 TGA profiles for a-Al (black lines) and m-Al (red lines) for untreated (solid lines) and pretreated (dashed lines) substrates obtained after direct synthesis and after reactive seeding (dot-dashed lines) employing pretreated substrates only. 


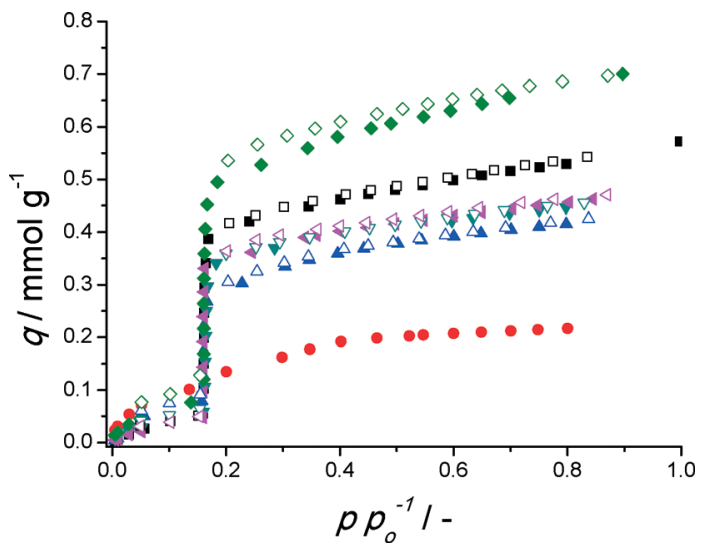

Fig. $7 \mathrm{H}_{2} \mathrm{O}$ adsorption isotherms $(298 \mathrm{~K})$ for direct synthesis on untreated $\mathrm{m}-\mathrm{Al}(\boldsymbol{\square})$ and a-Al $(O)$ and on pretreated m-Al (method A) $(\Delta)$ and a-Al (method B) $(\nabla)$ and for reactive seeding on pretreated m-Al $(\operatorname{method} A)(\zeta)$ and a-Al $(\operatorname{method} B)(\checkmark)$. Loading, $q$, is given per total mass of sample (substrate + MOF coating). Solid symbol adsorption, open symbol desorption branch.

the MOF structure. Hence further assumptions would be necessary, e.g. that all carbon atoms present in the sample belong to CAU-10-H on the substrate. As aluminium is predominantly present in the sample, because of the thickness of the dense support with respect to the porous coated layer, the uncertainty in the quantity of carbon present is anticipated to be large. Furthermore, weighing (dried) substrates before and after coating synthesis gives a skewed image as a-Al substrates are seemingly more prone to leaching and thus, for the same synthesis method, always display a lower weight of CAU-10-H (Table S1†). This method thus does not yield a clear indication on the amount of MOF present. Here, it is chosen to display trends in CAU-10-H based on two simple and rather unsophisticated methods, all with inherent drawbacks.

- First, one could estimate the amount of CAU-10-H on the substrate by the weight loss observed between 450 and $700{ }^{\circ} \mathrm{C}$, assuming that all the loss observed (Fig. 6) is caused by burning the organic ligand and subsequently that all decomposed ligands were incorporated in the CAU-10-H structure.

- Second, by stating that the step in water adsorption (Fig. 7) is solely caused by CAU-10-H on the substrate surface and assuming that this has the same specific adsorption capacity as bulk MOF powder (Fig. 1), one can calculate the amount of MOF present on the substrate surface.

This intrinsically means however that substrates need to be cut to smaller proportions be able to fit a sample holder for water adsorption $(\sim 8 \times 20 \mathrm{~mm})$ or TGA analysis $(\sim 8 \times$ $8 \mathrm{~mm})$. This procedure might thus cause some loss of MOF material on the cutting edges and underestimate the actual loading. Nonetheless, estimated MOF loadings of these analyses are shown in Table 1, per unit of substrate surface.

The MOF loadings determined by TGA and by water adsorption show similar trends and a fair agreement, although the quantity obtained by analysing TGA is always lower than for $\mathrm{H}_{2} \mathrm{O}$ adsorption. This could well be because of the necessity to cut these plates to manageable sizes. If this is the case, the loading indicated by $\mathrm{H}_{2} \mathrm{O}$ adsorption also underestimates the actual MOF loading, as the substrates have to be cut, but to lesser extent (vide supra). Nonetheless, based on water adsorption, a $25 \%$ increase in capacity has been achieved with reactive seeding on pretreated a-Al compared to the direct synthesis on untreated $\mathrm{m}$-Al, the protocol used in previous work. ${ }^{17}$ This means that up to $48 \mathrm{~kJ}$ of heat can be withdrawn in the evaporator of an AHP/ADC per square meter of coated anodized aluminium surface (for metallic aluminium this is only $38 \mathrm{~kJ}$ (ref. 17)). On top of that, reactive seeding leads to a more homogenous coverage of crystals and a significantly narrower crystal size distribution as well as enhanced purity of CAU-10-H.

Performance. Cyclic water ad- and desorption measurements were performed gravimetrically for both pure CAU-10-H (conventional synthesis) and coated CAU-10-H (reactive seeding on pretreated a-Al (method B)) to assess stability of the coated substrate and comparing the response times of both systems (Fig. 8).

Clearly both bulk CAU-10-H and the substrate do not lose adsorption capacity over nine cycles. For the bulk powder this is expected as Fröhlich et al. already demonstrated that adsorption capacity of CAU-10-H is retained over 700 cycles. ${ }^{14}$ Clearly, stability is not compromised when this MOF is coated on a substrate, a feature indispensable for application in adsorption driven heat pumps and chillers. No loss of coated CAU-10(Al)-H was observed (in powder form) after these cyclic measurements, a first indication that these coatings are indeed mechanically robust enough to withstand cyclic ad- and desorption measurements. Furthermore, the response of the amount adsorbed on a temperature step change is significantly more rapid for the coated sample, indicating that heat and mass transport are enhanced when a coated substrate is employed, in comparison to the powder sample. The loading, however, expressed per unit mass of sample, is significantly lower for the substrate than for the bulk powder. This is because the aluminium substrate has a thickness of $\sim 0.5 \mathrm{~mm}$, and its coating has only a thickness in the order of 60-120 $\mu \mathrm{m}$ (SEM images), so most of the sample mass comes from the bulk aluminium. For application in AHP/ADC devices a thermodynamic optimization analysis of the coating thickness with respect to the support thickness

Table 1 Indicative amounts of CAU-10-H present on selected substrates, estimated from TGA analysis and volumetric water adsorption

\begin{tabular}{llll}
\hline & & \multicolumn{2}{l}{ Loading $/ \mathrm{mg} \mathrm{cm}^{-2}$} \\
\cline { 3 - 4 } Synthesis method & Substrate & TGA & $\mathrm{H}_{2} \mathrm{O}$ ads. \\
\hline Direct, no pretreatment & $\mathrm{m}-\mathrm{Al}$ & 2.4 & 4.0 \\
& $\mathrm{a}-\mathrm{Al}$ & \\
Direct, with pretreatment & $\mathrm{m}-A l^{b}$ & 1.0 & n.d. \\
& $\mathrm{a}-\mathrm{Al}^{c}$ & 2.6 & 2.8 \\
Reactive seeding $^{d}$ & $\mathrm{~m}-\mathrm{Al}^{b}$ & 2.8 & 3.6 \\
& $\mathrm{a}-\mathrm{Al}^{c}$ & 2.8 & 3.3 \\
& & 4.4 & 5.0
\end{tabular}

${ }^{a}$ Without HCl. ${ }^{b}$ Method A. ${ }^{c}$ Method B. ${ }^{d}$ With pretreatment, seed reaction time 4 h. n.d. means not determinable. 

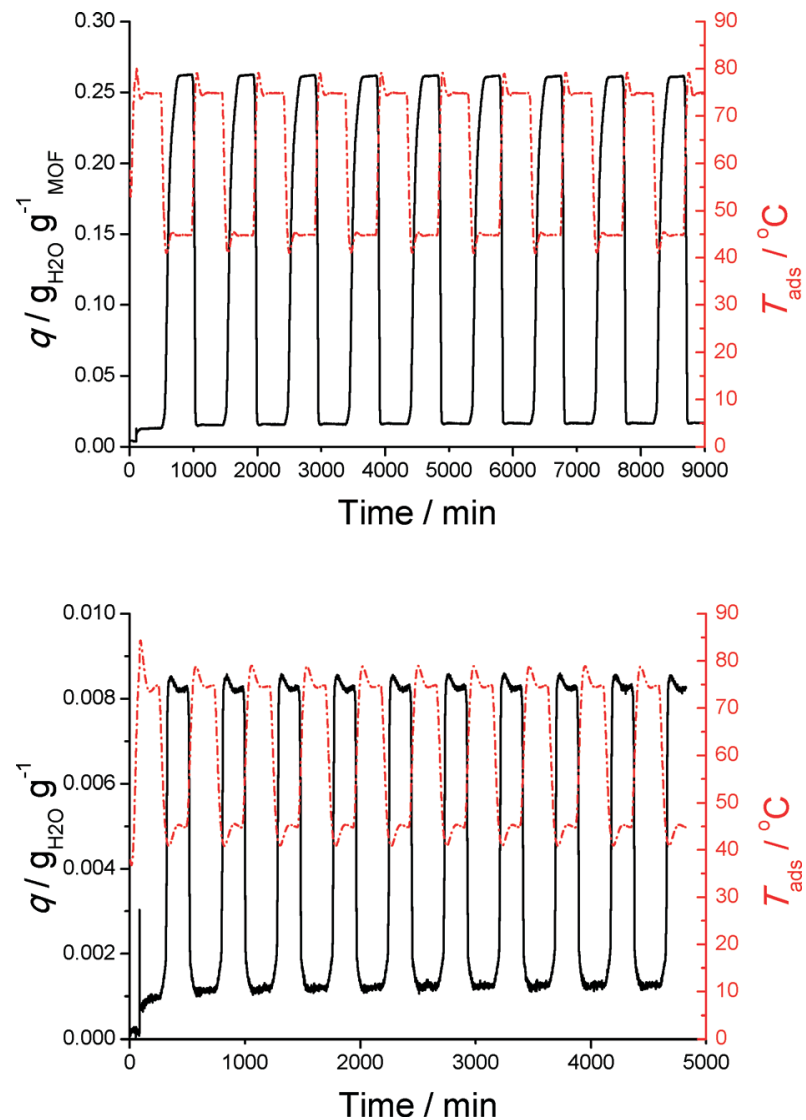

Fig. 8 Cyclic gravimetric water adsorption-desorption measurements upon temperature step changes between 45 and $75{ }^{\circ} \mathrm{C}$, at a fixed water vapour pressure (26 mbar) for bulk CAU-10-H powder (conventional synthesis, top) and $\mathrm{CAU}-10-\mathrm{H}$ synthesized on pretreated a-Al (method B) using reactive seeding (bottom). Adsorbed amount of water (left $y$-axis, black solid line) and temperature (right $y$-axis, red dashed line) both as function of time. Amount adsorbed is indicated per gram of total sample, including the mass of the support for the coated a-Al.

should be performed, considering not only capacity, but also heat and mass transport.

The optimal coating method found for CAU-10-H on metallic aluminium substrates requires a number of necessary steps starting from metallic aluminium: (i) anodization, (ii) pretreatment, (iii) reactive seeding and (iv) secondary growth, which may hamper scalability of these coatings. In this sense, it might be beneficial to integrate some of the steps, especially merging anodization and pretreatment should be feasible. Additionally, the employed anodized layer was selected because of commercial availability, not because it necessarily holds optimal properties for MOF attachment. Optimizing anodization conditions and combining this step with the seeding step might thus further improve the manufacture of CAU-10-H coatings.

\section{Conclusions}

For manufacturing of CAU-10-H coatings on aluminium substrates, syntheses on metallic aluminium (m-Al) with varying manufacture conditions did not result in a notable improved coating quality. Any deviation from the standard synthesis protocol (SSP) leads to worse coatings.

The porous amorphous aluminium oxide layer of anodized aluminium (a-Al) is more reactive and thus crystallization on the surface is easier. However, a significant amount of by-product(s) is formed, attributed to the higher content of extracted aluminium ions near the surface.

Substrate pretreatment improves both reproducibility and coating quality of CAU-10-H on both m-Al and a-Al substrates. For $\mathrm{m}$-Al substrates cleaning with acetone (method A) yields optimal results. For a-Al, additionally the substrate should be contacted with a diluted $\mathrm{HCl}$ solution (6\% in water) (method B) for optimal results. Despite the improvement achieved, obtained coatings are still suboptimal in coverage, homogeneity of crystal sizes and purity. Separation of crystal nucleation and growth yields significantly improved quality, showcased by the high purity and homogeneous crystal size distribution obtained by both thermal and reactive seeding on pretreated substrates. Especially reactive seeding in conjunction with pretreated a-Al (method B) yields full coverage of the substrate surface, a homogeneous layer thickness, narrow crystal size distribution, and high purity of the crystalline phase, fulfilling every requirement for application in adsorption driven heat pumps and chillers, namely: full coverage of the substrate surface, high MOF loading, homogeneous layer thickness, narrow crystal size distribution, sufficient stability and high purity of the crystalline phase. Coatings created with this method do not lose capacity upon repeated water adsorption-desorption cycles and respond much faster to temperature changes than bulk CAU-10-H powder.

\section{Acknowledgements}

Financial support from the Advanced Dutch Energy Materials (ADEM) program of the Dutch Ministry of Economic Affairs, Agriculture and Innovation is gratefully acknowledged.

\section{Notes and references}

1 M. F. De Lange, K. J. F. M. Verouden, T. J. H. Vlugt, J. Gascon and F. Kapteijn, Chem. Rev., 2015, (Submitted).

2 H. Reinsch, M. A. van der Veen, B. Gil, B. Marszalek, T. Verbiest, D. de Vos and N. Stock, Chem. Mater., 2012, 25, 17-26.

3 R. J. Sheehan, Ullmann's Encyclopedia of Industrial Chemistry, Wiley-VCH Verlag GmbH \& Co., 2000.

4 H. Kakiuchi, S. Shimooka, M. Iwade, K. Oshima, M. Yamazaki, S. Terada, H. Watanabe and T. Takewaki, Kagaku Kogaku Ronbunshu, 2005, 31, 361-364.

5 H. Kakiuchi, S. Shimooka, M. Iwade, K. Oshima, M. Yamazaki, S. Terada, H. Watanabe and T. Takewaki, Kagaku Kogaku Ronbunshu, 2005, 31, 273-277.

6 S. Shimooka, K. Oshima, H. Hidaka, T. Takewaki, H. Kakiuchi, A. Kodama, M. Kubota and H. Matsuda, J. Chem. Eng. Jpn., 2007, 40, 1330-1334. 
7 MITSUBISHI PLASTICS, Zeolitic Water Vapor Adsorbent: AQSOA, http://www.aaasaveenergy.com/products/001/pdf/ AQSOA_1210E.pdf, Accessed 17-01, 2015.

8 G. Liu, P. Tian, Y. Zhang, J. Li, L. Xu, S. Meng and Z. Liu, Microporous Mesoporous Mater., 2008, 114, 416-423.

9 Y. Xu, P. J. Maddox and J. W. Couves, J. Chem. Soc., Faraday Trans., 1990, 86, 425-429.

10 A. M. Prakash and S. Unnikrishnan, J. Chem. Soc., Faraday Trans., 1994, 90, 2291-2296.

11 R. D. Gougeon, E. B. Brouwer, P. R. Bodart, L. Delmotte, C. Marichal, J.-M. Chézeau and R. K. Harris, J. Phys. Chem. B, 2001, 105, 12249-12256.

12 F. Jiang, Z. Tang, J. Zhai, J. Ye and J. Han, Microporous Mesoporous Mater., 2006, 92, 129-133.

13 Y. Wan, C. D. Williams, C. V. Duke and J. J. Cox, J. Mater. Chem., 2000, 10, 2857-2862.

14 D. Fröhlich, S. K. Henninger and C. Janiak, Dalton Trans., 2014, 43, 15300-15304.

15 Y. K. Seo, J. W. Yoon, J. S. Lee, Y. K. Hwang, C. H. Jun, J. S. Chang, S. Wuttke, P. Bazin, A. Vimont and M. Daturi, Adv. Mater., 2012, 24, 806-810.

16 T. A. Nijhuis, A. E. Beers, T. Vergunst, I. Hoek, F. Kapteijn and J. A. Moulijn, Catal. Rev.: Sci. Eng., 2001, 43, 345-380.

17 M. De Lange, C. Ottevanger, M. Wiegman, T. Vlugt, J. Gascon and F. Kapteijn, CrystEngComm, 2015, 17, 281-285.

18 T. Ahnfeldt and N. Stock, CrystEngComm, 2012, 14, 505-511.

19 S.-N. Kim, J. Kim, H.-Y. Kim, H.-Y. Cho and W.-S. Ahn, Catal. Today, 2013, 204, 85-93.

20 N. A. Khan, I. J. Kang, H. Y. Seok and S. H. Jhung, Chem. Eng. J., 2011, 166, 1152-1157.

21 Y. Hu, X. Dong, J. Nan, W. Jin, X. Ren, N. Xu and Y. M. Lee, Chem. Commun., 2010, 47, 737-739.

22 V. V. Guerrero, Y. Yoo, M. C. McCarthy and H.-K. Jeong, J. Mater. Chem., 2010, 20, 3938-3943.

23 M. Arnold, P. Kortunov, D. J. Jones, Y. Nedellec, J. Kärger and J. Caro, Eur. J. Inorg. Chem., 2007, 2007, 60-64.

24 H. Bux, A. Feldhoff, J. Cravillon, M. Wiebcke, Y.-S. Li and J. Caro, Chem. Mater., 2011, 23, 2262-2269.

25 F. Schüth, K. S. W. Sing and J. Weitkamp, Handbook of porous solids, Wiley-VCH, 2002.

26 M. F. De Lange, T. J. H. Vlugt, J. Gascon and F. Kapteijn, Microporous Mesoporous Mater., 2014, 200, 199-215.

27 A. García Márquez, A. Demessence, A. E. Platero-Prats, D. Heurtaux, P. Horcajada, C. Serre, J.-S. Chang, G. Férey, V. A. de la Peña-O'Shea, C. Boissière, D. Grosso and C. Sanchez, Eur. J. Inorg. Chem., 2012, 2012, 5165-5174.
28 H.-Y. Cho, D.-A. Yang, J. Kim, S.-Y. Jeong and W.-S. Ahn, Catal. Today, 2012, 185, 35-40.

29 H. Furukawa, F. Gándara, Y.-B. Zhang, J. Jiang, W. L. Queen, M. R. Hudson and O. M. Yaghi, J. Am. Chem. Soc., 2014, 136, 4369-4381.

30 J. Reboul, S. Furukawa, N. Horike, M. Tsotsalas, K. Hirai, H. Uehara, M. Kondo, N. Louvain, O. Sakata and S. Kitagawa, Nat. Mater., 2012, 11, 717-723.

31 T. Campbell, R. K. Kalia, A. Nakano, P. Vashishta, S. Ogata and S. Rodgers, Phys. Rev. Lett., 1999, 82, 4866-4869.

32 P. P. Mardilovich, A. N. Govyadinov, N. I. Mukhurov, A. M. Rzhevskii and R. Paterson, J. Membr. Sci., 1995, 98, 131-142.

33 E. Stavitski, M. Goesten, J. Juan-Alcañiz, A. Martinez-Joaristi, P. Serra-Crespo, A. V. Petukhov, J. Gascon and F. Kapteijn, Angew. Chem., Int. Ed., 2011, 50, 9624-9628.

34 E. Biemmi, S. Christian, N. Stock and T. Bein, Microporous Mesoporous Mater., 2009, 117, 111-117.

35 H. Reinsch and N. Stock, Microporous Mesoporous Mater., 2013, 171, 156-165.

36 Y.-B. Dong, Y.-Y. Jiang, J. Li, J.-P. Ma, F.-L. Liu, B. Tang, R.-Q. Huang and S. R. Batten, J. Am. Chem. Soc., 2007, 129, 4520-4521.

37 Y. X. Sun and W. Y. Sun, Chin. Chem. Lett., 2014, 25, 823-828.

38 J. W. Muller, Crystallization, Butterworth-Heinemann Ltd, Oxford, Revised edn., 2001, vol. 4.

39 H. Guo, G. Zhu, I. J. Hewitt and S. Qiu, J. Am. Chem. Soc., 2009, 131, 1646-1647.

40 R. Makiura, S. Motoyama, Y. Umemura, H. Yamanaka, O. Sakata and H. Kitagawa, Nat. Mater., 2010, 9, 565-571.

41 K. Yusenko, M. Meilikhov, D. Zacher, F. Wieland, C. Sternemann, X. Stammer, T. Ladnorg, C. Wöll and R. A. Fischer, CrystEngComm, 2010, 12, 2086-2090.

42 F. Jeremias, D. Frohlich, C. Janiak and S. K. Henninger, RSC Adv., 2014, 4, 24073-24082.

43 S. Hermes, D. Zacher, A. Baunemann, C. Wöll and R. A. Fischer, Chem. Mater., 2007, 19, 2168-2173.

44 I. Bezverkhyy, G. Ortiz, G. Chaplais, C. Marichal, G. Weber and J.-P. Bellat, Microporous Mesoporous Mater., 2014, 183, 156-161.

45 K. L. Nagy and A. C. Lasaga, Geochim. Cosmochim. Acta, 1992, 56, 3093-3111.

46 J. Y. Bottero, J. M. Cases, F. Fiessinger and J. E. Poirier, J. Phys. Chem., 1980, 84, 2933-2939.

47 A. Boumaza, L. Favaro, J. Lédion, G. Sattonnay, J. B. Brubach, P. Berthet, A. M. Huntz, P. Roy and R. Tétot, J. Solid State Chem., 2009, 182, 1171-1176. 